\title{
CARACTERIZACIÓN MORFOLÓGICA DE TRECE VARIEDADES DE OLIVO (Oleo europaea L.) INTRODUCIDAS EN EL GERMOPLASMA DEL INPREX - TACNA
}

\section{RESUMEN}

El presente trabajo tuvo como propósito describir la morfologia de algunas variedades de olivo (Olea europaea L.) del germoplasma INPREX-UNJBG-Tacna, para disponer un banco de datos que servirá para trabajos de investigación en programas de mejoramiento genético.Para la identificación y descripción, se han considerado 28 caracteres botánicos, correspondiendo: 5 de hojas, 4 de inflorescencias, 10 de fruto y 9 de endocarpio, propuestos por Barranco y Rallo (1984).

Se determinó que las 13 entradas presentan morfologia variable en cuanto a: Forma de limbo, tamaño de limbo, relación Iongitud/anchura, color de haz. Estructura de Inflorescencia, forma de inflorescencia, presencia de flores supernumerarias. Presencia de lenticelas en el fruto, forma del fruto, simetria del fruto, forma de ápice del fruto, cavidad peduncular. Forma del endocarpio, simetria del endocarpio, tamaño del endocarpio, posición del diámetro transversal máximo, superficie, número de surcos fibro vasculares y terminación del ápice.
Responsable : Ing. Rodi David Alférez Garcia Miembro : : Ing. Magno Santos Robles Tello

\section{INTRODUCCIÓN}

La olivicultura en el Perú se inicia en la costa central el año de 1560 con la introducción de plantones procedentes de Sevilla, España (Echeandia, 1929; De la Torre, 1953).

A partir de aquel año se difundió su cultivo hacia el norte y el sur, habiendo encontrado su óptimo ecológico para el crecimiento y desarrollo en la región sur de nuestro país. La superficie cultivada de olivos (Olea europaea $L$ ) total mundial alcanza 8885450 ha. (COI, 1999). El Perú cuenta con 8000 ha cultivadas (FAO, 2005), además ocupa el cuarto lugar en el ranking de rendimiento mundial con 4,9 TM/ha. (FAO, 2003). La región de Tacna cuenta con 5846 ha de olivo, con rendimientos de $6514 \mathrm{~kg} / \mathrm{ha}$ (Anuario Estadistico Regional 2004-MINAG).La olivicultura en las irrigaciones de "La Yarada", "Los Palos", "Magollo" y "Sama", ubicados en la región de Tacna, conducidas bajo tecnologia media y avanzada, constituyen una actividad agricola rentable. Este hecho ha despertado un gran interés en los agricultores, por ampliar la frontera agricola e introducir nuevas variedades para mesa y aceite.

Por los antecedentes mencionados, se han iniciado estudios sobre el comportamiento de nuevas variedades introducidas a nuestro pais. En tal sentido, se ha planteado el siguiente objetivo: Hacer una descripción morfológica de las hojas, flores, frutos y endocarpios de 13 entradas de olivo (Olea europaea L.) implementadas en el germoplasma del INPREX-UNJBG-Tacna.

\section{MATERIALYMÉTODOS}

\section{1) Ubicación del Campo Experimental}

El presente proyecto de investigación se ejecutó en el Instituto de Investigación, Producción y Extensión de la Universidad Nacional Jorge Basadre Grohmann, ubicado en el distrito, provincia y departamento de Tacna, bajo las coordenadas: L.S. $18^{\circ} 1^{\prime}$, L.W. $71^{\circ} 15^{\prime}$ y altitud de $560 \mathrm{msnm}$.

Material experimental plantel de árboles de olivo de trece variedades (cuadro № 1); Catálogo descriptor; Cámara fotográfica digital; Papel milimetrado; Regla; Bolsas de polietileno; Tijera de podar; Balanza y Termómetro.

Cuadro $N^{\circ}$ 01: Variedades de olivo (Olea europaea L.) en estudio

\begin{tabular}{|c|c|c|c|}
\hline $\begin{array}{c}\mathbf{N}^{\mathbf{0}} \\
\text { Tratamiento }\end{array}$ & Variedad & Propósito & Origen \\
\hline 01 & Sevillana (clon) & Mesa & Perú \\
\hline 02 & Sevillana (injertada) & Mesa & Perú \\
\hline 03 & Limoncillo & Mesa & España \\
\hline 04 & Cornezuelo & Aceite & España \\
\hline 05 & Konservolia & Mesa/aceite & Grecia \\
\hline 06 & Uovo di piccione & Mesa & Italia \\
\hline 07 & Manzanilla de Sevilla & Mesa & España \\
\hline 08 & Campanil & Mesa & España \\
\hline 09 & Azapa & Mesa/aceite & Chile \\
\hline 10 & Azeradj & Mesa/aceite & Argelia \\
\hline 11 & Picholine & Mesa/aceite & Francia \\
\hline 12 & Arauco & Mesa & Argentina \\
\hline 13 & Leccino & Aceitera & Italia \\
\hline
\end{tabular}




\section{2) Variables evaluadas}

Hoja (forma, tamaño, relación L/A, brillo del haz, color del haz); Inflorescencia (estructura, forma, flores supernumerarias, grosor de botones); Frutos (color a la maduración, presencia de lenticelas, forma, simetria, tamaño, relación pulpa, posición del diámetro máximo, forma del ápice, posición punto estilar, cavidad peduncular, sección transversal máxima); Endocarpio (forma, simetria, tamaño, posición diámetro transversal máximo, superficie, número de surcos, distribución de surcos, forma de ápice, terminación de ápice).
Se han utilizado descriptores propuestos por Barranco y Rallo (1984). En cada carácter se distinguen las diferentes categorias en las que se pueden clasificar los cultivares

\section{RESULTADOS}

Los resultados se obtuvieron de una muestra de 30 hojas, inflorescencias, frutos y endocarpios, tomados de 3 árboles de cada tratamiento, de cuatro cuadrantes $(E, O, N, S)$ en las 13 entradas de olivo, provenientes del germoplasma INPREX - UNJBG - 2006/2007 - Tacna.

Tabla № 01: Caracterización morfológica de las hojas del olivo (Olea europaea L.)

\begin{tabular}{|c|c|c|c|c|c|}
\hline $\begin{array}{c}\mathbf{N}^{\circ} \\
\text { Tratam. }\end{array}$ & Forma del limbo & Tamaño del limbo & $\begin{array}{c}\text { Relación } \\
\text { longitud/anchura }\end{array}$ & $\begin{array}{c}\text { Brillo del } \\
\text { haz }\end{array}$ & $\begin{array}{c}\text { Color del } \\
\text { haz }\end{array}$ \\
\hline 1 & Lanceolada & Muy grande & Larga y estrecha & Mate & Verde claro \\
\hline 2 & Eliptico-lanceolada & Muy grande & Larga y estrecha & Mate & Verde oscuro \\
\hline 3 & Lanceolada & Muy grande & Larga y estrecha & Mate & Verde claro \\
\hline 4 & Lanceolada & Muy grande & Larga y estrecha & Mate & Verde claro \\
\hline 5 & Lanceolada & Muy grande & Corta y estrecha? & Mate & Verde claro \\
\hline 6 & Eliptico-lanceolada & ?Muy grande? & Larga y ancha & Mate & Verde claro \\
\hline 7 & Eliptico-lanceolada & Muy grande? & Corta y estrecha & Mate? & Verde claro \\
\hline 8 & Lanceolada & Muy grande & Larga y estrecha & Mate & Verde claro \\
\hline 9 & Lanceolada & Muy grande & Larga y estrecha? & Mate & Verde oscuro \\
\hline 10 & Lanceolada & Muy grande & Larga y estrecha & Mate & Verde oscuro \\
\hline 11 & Lanceolada & Muy grande & Corta y estrecha & Mate & Verde oscuro \\
\hline 12 & Lanceolada & Muy grande & Larga y estrecha & Mate & Verde oscuro \\
\hline 13 & Lanceolada & Grande & Larga y estrecha & Mate & Verde oscuro \\
\hline
\end{tabular}

Tabla № 02: Caracterización morfológica de inflorescencias del olivo (Olea europaea L.)

\begin{tabular}{|c|c|c|c|c|}
\hline $\begin{array}{c}N^{0} \\
\text { Tratam. }\end{array}$ & Estructura & Forma & $\begin{array}{c}\text { Presencia de flores } \\
\text { supernumerarias }\end{array}$ & $\begin{array}{c}\text { Grosor de } \\
\text { botones }\end{array}$ \\
\hline 1 & Larga y compacta & Paniculada & Ausente & Mediano \\
\hline 2 & Larga y compacta & Paniculada & Ausente & Mediano \\
\hline 3 & Larga y compacta & Paniculada & Ausente & Mediano \\
\hline 4 & Corta y laxa & Paniculada espiciforme & Ausente & Pequeño \\
\hline 5 & Corta y laxa & Paniculada espiciforme & Ausente & Mediano \\
\hline 6 & Corta y laxa & Racemosa & Ausente & Mediano? \\
\hline 7 & Larga y laxa & Paniculada & Ausente & Mediano \\
\hline 8 & Larga y laxa & Paniculada & Ausente & Mediano \\
\hline 9 & Larga y laxa & Paniculada espiciforme & Ausente & Mediano \\
\hline 10 & Corta y laxa & Paniculada & Presente & Mediano \\
\hline 11 & Corta y compacta & Paniculada & Ausente & Pequeño \\
\hline 12 & Larga y compacta & Paniculada & Ausente & Mediano \\
\hline 13 & Larga y compacta & Paniculada & Ausente & \\
\hline
\end{tabular}


Tabla N 03a: Evaluación morfológica de frutos de olivo (Olea europaea L.) INPREX - 2007/04/20

\begin{tabular}{|c|c|c|c|c|c|}
\hline $\begin{array}{c}\mathbf{N}^{0} \\
\text { Tratam. }\end{array}$ & Color & Lenticelas & Forma & Simetria & Tamaño \\
\hline 1 & Negro & Visibles & Alargada & Ligeramente asimétrica & Grande \\
\hline 2 & Negro & Visibles & Alargada & Ligeramente asimétrica & Grande \\
\hline 3 & Negro & Visibles & Ligeramente alargada & Asimétrica & Grande \\
\hline 4 & Negro & Visibles & Alargada & Ligeramente asimétrica & Grande \\
\hline 5 & Negro & Visibles & Ligeramente esferoidal & Ligeramente asimétrica & Grande \\
\hline 6 & Negro & No Visibles & Ligeramente alargada & Simétrica & Grande \\
\hline 7 & Negro & Visibles & Ligeramente esferoidal & Simétrica & Grande \\
\hline 8 & Negro & Visibles & Alargada & Ligeramente asimétrica & Grande \\
\hline 9 & Negro & Visibles & Ligeramente alargada & Ligeramente asimétrica & Grande \\
\hline 10 & Negro & Visibles & Alargada & Ligeramente asimétrica & Pequeño \\
\hline 11 & Negro & Visibles & Alargada & Ligeramente asimétrica & Grande \\
\hline 12 & Negro? & Visibles? & Ligeramente alargada & Ligeramente asimétrica & Grande? \\
\hline 13 & Negro & Visibles & Alargada & Ligeramente asimétrica & Grande \\
\hline
\end{tabular}

Tabla Nº3b: Evaluación morfológica de frutos de olivo (Olea europaea L.) INPREX - 2007/04/20

\begin{tabular}{|c|c|c|c|c|c|}
\hline $\begin{array}{c}\text { No } \\
\text { Tratam. }\end{array}$ & $\begin{array}{c}\text { Posición } \\
\text { máximo }\end{array}$ & $\begin{array}{c}\text { Forma del } \\
\text { Apice }\end{array}$ & $\begin{array}{c}\text { Posición punto } \\
\text { estilar }\end{array}$ & $\begin{array}{c}\text { Cavidad } \\
\text { peduncular }\end{array}$ & $\begin{array}{c}\text { Sección transversal } \\
\text { máxima }\end{array}$ \\
\hline 1 & Centrado & Apuntado & Desplazado & Amplia & Circular \\
\hline 2 & Centrado & Apuntado & Desplazado & Angosta & Circular \\
\hline 3 & Centrado & Con pezón & Desplazado & Angosta & Circular \\
\hline 4 & Centrado & Apuntado & Desplazado & Angosta & Circular \\
\hline 5 & Centrado & Redondeado & Desplazado & Angosta & Circular \\
\hline 6 & Centrado & Redondeado & Desplazado & Angosta & Circular \\
\hline 7 & Centrado & Redondeado & Desplazado & Amplia & Circular \\
\hline 8 & Centrado & Apuntado & Desplazado & Angosta & Circular \\
\hline 9 & Centrado & Apuntado & Desplazado & Amplia & Circular \\
\hline 10 & Centrado & Apuntado & Desplazado & Angosta & Circular \\
\hline 11 & Centrado & Apuntado & Desplazado & Angosta & Circular \\
\hline 12 & Centrado & Apuntado & Desplazado & Amplia & Circular \\
\hline 13 & Centrado & Apuntado & Desplazado & Angosta & Circular \\
\hline
\end{tabular}

Tabla N 04a: Evaluación morfológica de endocarpios de frutos de olivo (Olea europaea L.) INPREX - 2007/05/13 INPREX - 2007/04/20

\begin{tabular}{|c|c|c|c|c|c|}
\hline $\begin{array}{c}\mathbf{N}^{0} \\
\text { Tratam. }\end{array}$ & Forma (A) & Simetría (A) & Tamaño & $\begin{array}{c}\text { Posición } 6 \\
\text { transversal máximo }\end{array}$ & Superficie \\
\hline 1 & Alargada & Asimétrica & Grande & Centrado & Rugosa \\
\hline 2 & Alargada & Asimétrica & Grande & Centrado & Rugosa \\
\hline 3 & Alargada & Ligeramente asimétrica & Grande & Centrado & Rugosa \\
\hline 4 & Alargada & Asimétrica & Grande & Centrado & Lisa \\
\hline 5 & Alargada & Ligeramente asimétrica & Grande & Centrado & Lisa \\
\hline 6 & Eliptica & Ligeramente asimétrica & Grande & Centrado & Rugosa \\
\hline 7 & Ovoidal & Ligeramente asimétrica & Grande & Centrado & Rugosa \\
\hline 8 & Ligeramente alargada & Ligeramente asimétrica & Grande & Centrado & Rugosa \\
\hline 9 & Ligeramente alargada & Ligeramente asimétrica & Grande & Centrado & Rugosa \\
\hline 10 & Ligeramente alargada & Ligeramente asimétrica & Mediano & Centrado & Lisa \\
\hline 11 & Alargada & Asimétrica & Grande & Centrado & Rugosa \\
\hline 12 & Alargada & Ligeramente asimétrica & Grande & Centrado & Rugosa \\
\hline 13 & Alargada & Ligeramente asimétrica & Grande & Hacia el ápice & Rugosa \\
\hline
\end{tabular}


Tabla № 4b: Evaluación morfológica de endocarpios de frutos de olivo (Olea europaea L.) INPREX - 2007/05/13

\begin{tabular}{|c|c|c|c|c|}
\hline $\begin{array}{c}\mathbf{N}^{0} \\
\text { Tratam. }\end{array}$ & $\begin{array}{l}\text { Número surcos } \\
\text { Fibro vasculares }\end{array}$ & $\begin{array}{l}\text { Distribución surcos } \\
\text { fibro vasculares }\end{array}$ & $\begin{array}{c}\text { Forma del } \\
\text { ápice }\end{array}$ & $\begin{array}{l}\text { Terminación } \\
\text { del ápice }\end{array}$ \\
\hline 1 & $7-10$ & Agrupada & Apuntada & Con mucrón \\
\hline 2 & $7 \cdot 10$ & Agrupada & Apuntada & Con mucrón \\
\hline 3 & $7-10$ & Agrupada & Apuntada & Con mucrón \\
\hline 4 & $>10$ & Agrupada & Apuntada & Con mucrón \\
\hline 5 & $7 \cdot 10$ & Agrupada & Apuntada & Con mucrón \\
\hline 6 & $7-10$ & Agrupada & Apuntada & Sin mucrón \\
\hline 7 & $>10$ & Agrupada & Apuntada & Sin mucrón \\
\hline 8 & $>10$ & Agrupada & Redondeada & Con mucrón \\
\hline 9 & $7-10$ & Agrupada & Apuntada & Con mucrón \\
\hline 10 & $7 \cdot 10$ & Agrupada & Apuntada & Con mucrón \\
\hline 11 & $7-10$ & Agrupada & Apuntada & Con mucrón \\
\hline 12 & $<7$ & Agrupada & Apuntada & Sin mucrón \\
\hline 13 & $<7$ & Agrupada & Apuntada & Sin mucrón \\
\hline
\end{tabular}

\section{CONCLUSIONES}

1. Doce variedades de olivo (Olea europaea L.) del INPREX- Tacna: Sevillana, Limoncillo, Cornezuelo, Konservolia, Uovo di piccione Manzanilla, Campanil, Azapa, Azeradj, Picholine, Arauco y Leccino son genotipos diferentes en sus características morfológicas engeneral.

2. El tamaño de hojas de los genotipos en estudio son de muy grande a grande, hecho que puede influir en una mayor capacidad fotosintética.

3. En cuanto al fruto, todos son de color negro y tamaño grande, excepto la variedad Azeradj que es pequeño. Todos son para mesa o doble propósito, exonerando a Leccino que es para aceite.

4. El tamaño del endocarpio (hueso): todos son grandes, excepto la variedad Azeradj, hecho que no favorecería a un mayor volumen del mesocarpio (pulpa) de la aceituna

\section{BIBLIOGRAFÍA}

Barranco D.; Fernández Escobar R.; Rallo, L. 1998. El Cultivo del Olivo. Ed. Mundi-Prensa, Madrid, $651 \mathrm{pp}$.

Caballero J. ; Del Río C. 1995 .Las Nuevas Plantaciones del Olivo. Córdoba.

Cavagnaro, P. 2001. Discriminación de variedades de olivo a través del uso de caracteres morfológicos y de marcadores moleculares.

AGRISCIENTIA, vol. XVIII: 27-35. Argentina.
Http://crean.org.ar/agriscientia/volumenes/

re Sumen/volumen\%2018/cavagnaro.pdf

Consejo Oleícola Internacional. 1996.

Enciclopedia Mundial del Olivo. Madrid, 479 pp.

De la Torre, 1953. Algunos Problemas Relacionados con la Propagación del Olivo. Tesis Ingeniero Agrónomo. Escuela Nacional de Agricultura. Lima.

Echeandia, C. 1929. El cultivo del olivo en Moquegua. Tesis Ingeniero Agrónomo,Escuela Nacional de Agricultura. Lima.

Floreal, D. 1976. Olivicultura.Madrid.281 pp.

Guerrero, A, 1997. Nueva Olivicultura.Madrid.128pp

Huanuco J. 2001. Caracterización Morfológica de las variedades de olivo en los departamentos de Moquegua y Tacna. Tesis Pre-grado. UNJBG. Tacna

Loussert, R; Brousse, G. 1980. El Olivo, Madrid517pp.

Sandoval V. Fuentealba C. ; Zamora S. 2006. Caracterización de la Biocenosis del Olivo, Olea europaea L. En el valle de Azapa. Arica, Chile.

Sotomayor, E. 2002. Propagación de olivo (Olea europaea L) CV. Apaza, por estaquillas semileñosas bajo diferentes medios de enraizamiento, por injertación de taller y estados tipo - fenológicos de diez vultivares del Banco de Germoplasma del valle de Azapa. Tesis ingeniero agrónomo. Tacna, Perú.

Tous,J; Romero, A. 1993. Variedades del Olivo. Barcelona. $172 \mathrm{pp}$.

University of California. 1985. Olive. California.148pp. 